\title{
A system for High-Resolution Face Scanning Based on Polarized Spherical Illumination
}

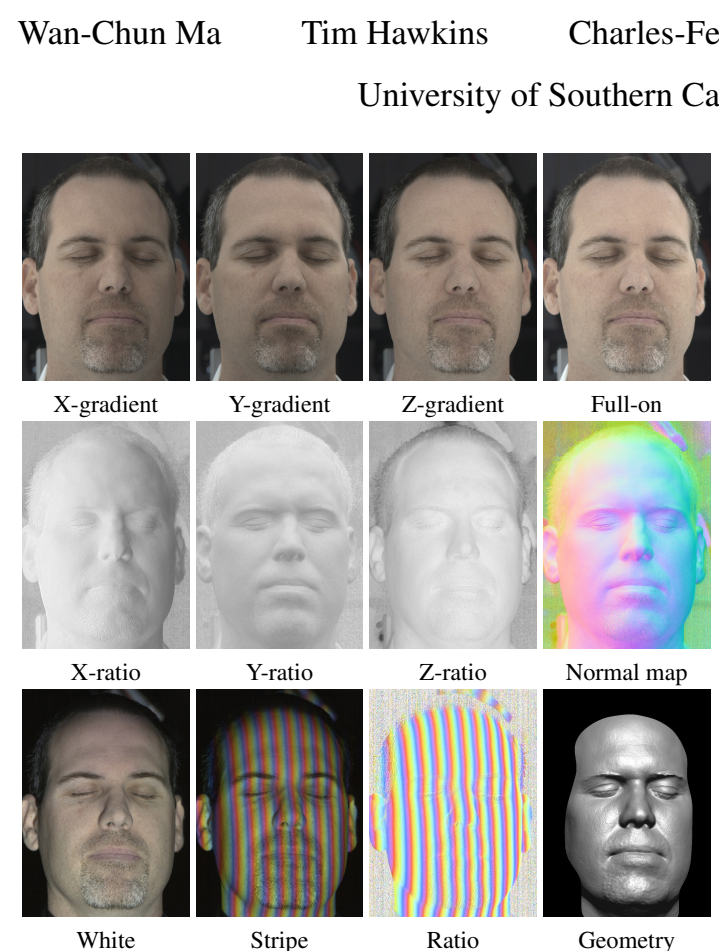

Figure 1: Top, images taken under polarized spherical gradient illumination from one polarization direction (i.e. diffuse only). Middle, corresponding ratio images and acquire normal maps. Bottom, projected full white pattern, one of the stripe patterns, corresponding ratio image, and acquired mesh (after optimization).

We developed a high-resolution face scanning system based on a new photometric stereo technique and multi-view stereo. We use a set of spherical gradient illumination patterns, along with linear polarization, to estimate normal maps of subjects from both diffuse and specular reflections separately. A structured-light-assisted twoview stereo system is employed to acquire 3D positions of the subject. This geometry is enhanced using normals from specular reflection, which reveal the true details of the surface, even for translucent objects for which laser-based scanning techniques would produce blurred results. Our technique is purely photometric, without any casting or painting process. The processing of the spherical illumination patterns is described in [Ma et al. 2007]. This sketch describes the key hardware components of the system.

Lighting apparatus for spherical gradient illumination. The key component of our system is an enhanced version of the Lightstage 5 device presened in [Wenger et al. 2005]. For our polarized gradient illumination conditions, we added the ability to simulate grayscale illumination values and fitted each lightsource with a carefully aligned linear polarizer. We simulate graycodes by switching the LED's on and off at a rate of $8 \mathrm{kHz}$. The modulation pattern is determined by a temporal error diffusion algorithm.

Camera setup. To capture the fine details of human skin we use two Canon 5D digital SLR cameras with $200 \mathrm{~mm}$ lenses. These cameras can burst about 14 images to internal RAM at a rate of 2.4 frames per second. With our optimized sequence of 13 images we are thus able to do a complete capture in just over five seconds. This high speed is important for minimizing subject motion.

Only one of the two cameras is used for normal and reflectance estimations; the other camera is used only for stereo recovery of
Mark Bolas Pieter Peers Paul Debevec

nia Centers of Creative Technologies

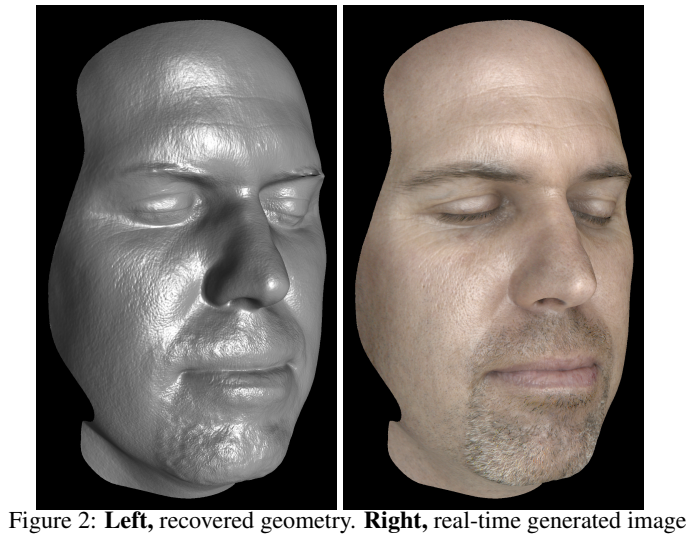

geometry. A linear polarizer is mounted on a servomotor in front of the master camera lens. The servomotor rotates the filter around an axis $45^{\circ}$ inclined from the axis of polarization, allowing the polarizer to be rapidly flipped between horizontal and vertical polarization to keep or cancel out specular reflections.

Structured-light-assisted two-view stereo system. The two cameras view the subject while a projector placed between them projects a sequence of four multi-frequency stripe patterns $P_{i}$ and one uniform white pattern $W$. Correspondences are computed between the cameras by finding best matches of the ratios $\frac{P_{i}}{W}$. This method has the benefit of dividing out the BRDF, leaving only global illumination as well as camera noise and non-linearity as sources of error. The biasing effects of global illumination are minimized by employing only relatively high-frequency patterns.

The projector itself uses a long throw lens to concentrate the available light and pixel resolution on the face of the human subject. We use a 3 LCD projector, which has two channels vertically polarized and one horizontally polarized. To get all three colors balanced and vertically polarized, we bank the projector $45^{\circ}$ and place a vertical polarizer in front. This will preserve $70.7 \%$ of the light, better than the $50 \%$ which would be maintained in the case of a vertical polarizer in front of a DLP projector.

For structured light image correspondence we then use only images captured with horizontal camera polarization, which exhibit very little specularity. Although in principle the BRDF is divided out in the ratio images, removing the specularity reduces sensitivity saturation and other camera response non-linearities.

The high-frequency detail available in the specular normals is added to the structured light scan as in [Nehab et al. 2005].

\section{References}

Ma, W.-C., Hawkins, T., Chabert, C.-F., Piers, P., Bolas, M., Weiss, M., AND DEBEVEC, P. 2007. Rapid acquisition of specular and diffuse normal maps from polarized spherical gradient illumination. Submitted to EGSR 2007.

Nehab, D., Rusinkiewicz, S., Davis, J., And Ramamoorthi, R. 2005. Efficiently combining positions and normals for precise $3 \mathrm{~d}$ geometry. ACM Transactions on Graphics 24, 3 (Aug.), 536-543.

Wenger, A., Gardner, A., Tchou, C., Unger, J., Hawkins, T., And DeBEVEC, P. 2005. Performance relighting and reflectance transformation with timemultiplexed illumination. ACM Transactions on Graphics 24, 3 (Aug.), 756-764. 